\title{
The Second Conference of the Romanian Society of Immuno-Dermatology, Bucharest, September 27-29, 2018
}

\author{
DANIEL BODA $^{1}$ and ARISTIDIS TSATSAKIS ${ }^{2}$ \\ ${ }^{1}$ Dermatology Research Laboratory, 'Carol Davila' University of Medicine and Pharmacy, 050474 Bucharest, Romania; \\ ${ }^{2}$ Deparment of Medicine, Laboratory of Toxicology, University of Crete, Heraklion 71003, Greece
}

Received November 29, 2018; Accepted December 6, 2018

DOI: 10.3892/etm.2018.7067

Immunological pathways have been extensively studied in the past 30 years as key processes of pathogenesis and outcome in several dermatological diseases and linked to new therapeutic treatment options. Thus, this special issue focuses on reviews, original articles and case presentations in immuno-dermatology, in order to partially elucidate those pathways and processes.

The main dermatological diseases covered in this special issue were immunological phenomena involved in psoriasis, lichen rubra, vitiligo, atopic dermatitis, chronic venous insufficiency and adverse drug reactions.

The wound healing process comprises a complex network of cells and molecules that are regulated in order to pursue tissue regeneration. The study by Bucur et al (1) focused on the capacity of alveolar blood clots, platelet-rich fibrin and plasma rich in growth factors (PRGF) to induce in vitro fibroblasts proliferation and migration as a measure of alveolar regeneration.

Rusu et al (2) aimed to analyze the interactions between $\mathrm{T}$ lymphocytes and a resorbable three-dimensional collagen matrix routinely used for soft tissue regeneration during periodontal surgery. The presented results showed that the main interaction between the collagen matrix and immune cells stimulated the activation of $\mathrm{T}$ cells and did not impair the healing process.

Grigore et al (3) analyzed the role of stress in the modulation of skin neurogenic inflammation in healthy volunteers. The results showed that exposure to stress is associated with an amplification of the mechanisms involved in capsaicin-induced skin neurogenic inflammation.

Correspondence to: Dr Daniel Boda, Dermatology Research Laboratory, 'Carol Davila' University of Medicine and Pharmacy, 8 Eroii Sanitari Avenue, 050474 Bucharest, Romania

E-mail: danielboday@yahoo.com

Key words: immunology, dermatology, Romanian Society
Ilie et al (4) concentrated their research on the in vivo confocal laser scanning microscopy (CLSM) imaging of skin inflammation: clinical applications and research directions, describing the principles of the in vivo CLSM technique, its role in the diagnosis and monitoring of inflammatory skin diseases, as well as some promising research directions to examine the dynamics of skin inflammation using this method.

Depicting the link between related purine derivatives and renal failure, Nicolae et al (5) conducted a prospective study on a group of psoriasis vulgaris patients and presented a series of positive and negative associations between purine derivatives and renal parameters. Based on their findings, they consider that severe psoriasis is a risk factor for the development of renal disease.

The original article of Boca et al (6) studied new potential therapeutic drugs in psoriasis, using an extract of sea buckthorn that shows promising results as an adjuvant or a component in psoriasis care protocols.

Although at present biological treatment constitutes the first-line treatment in moderate to severe forms of psoriasis, methotrexate (MTX) is an important anti-inflammatory and anti-proliferative drug.

Balanescu et al (7) demonstrated the effects of MTX on cardiovascular adverse reaction in immune-mediated dermatological conditions stating that the cardiovascular effects of MTX, albeit incompletely understood, are explained by its antiproliferative, immunosuppressive, anti-inflammatory and antiatherogenic effects.

Surcel et al (8) examined in a research article the Imiquimod-based murine model of psoriatic dermatitis as an alternative to traditional models of experimental psoriasis in mice and the induced dermatitis closely mimicking the pathologic changes in human psoriasis.

Focusing on new treatment options in vitiligo using bioactive compounds of Piper nigrum (PN), Mihaila et al (9) showed that the PN extract and its main alkaloid, piperine, promote melanocyte proliferation in vivo, which is consistent with previous research.

In the pathogenesis of lichen planus (LP) the link between this chronic skin condition and related pathologies has often been encountered. The review of Georgescu et al (10) aimed 
to summarize the main potential mechanisms involved in the association between LP and HCV infection. Understanding the link between the two disorders may shed some light on the pathogenesis of LP, which is a challenging issue.

In the same pathology of lichen rubra, Ianosi et al (11) focused on new in vivo diagnostic tools in order to properly quantify this condition and avoid unnecessary biopsies. At present, classical invasive diagnostic methods are replaced by modern non-invasive techniques, such as dermoscopy, reflectance confocal microscopy, optical coherence tomography, ultrasound and diffuse reflection spectrophotometry.

A broad number of patients suffer from atopic dermatitis, the most commonly accepted prevalence of atopic patients being approximately $10 \%$ of the total population. Deleanu et al (12) reviewed biological treatment in atopic dermatitis with a focus on novel targeted agents: systemic immunotherapy (Omalizumab, Dupilumab, Lebrikizumab, Tralokinumab, Nemolizumab, Ustekinumab, Fezakinumab, Tezepelumab, Apremilast, and allergen-specific immunotherapy), and topical agents (Tofacitinib, Crisaborole).

Nedelea et al (13) reviewed the clinical features and etiology of isolated angioedema. Emphasizing that mast cell-mediator release is frequently observed, the authors of that study elucidated that the clinical pattern and the possible causes of isolated angioedema are the key to a correct diagnosis.

Sabau et al (14) focused on new diagnostic procedures in the diagnosis of atopic dermatitis (AD) using high frequency ultrasonography and the results indicate that skin ultrasonography is able to assess specific modifications of the AD skin.

Solomon et al (15) concentrated their study on reviewing the influence of lifestyle factors on the outcome of AD as a multifactorial chronic inflammatory disease with an incompletely understood etiopathogenesis.

The link between life style factors and autoimmune diseases was also shown by Constantin et al (16) in a review on lupus erythematosus patients. The study clearly emphasizes the beneficial role of personalized diet in patients with SLE, and the information presented could be used in daily practice.

Chronic Venous Disease (CVD) affects millions of individuals, negatively influencing the patient's quality of life (QoL), with most of the patients being diagnosed with CVD in advanced stages. Branisteanu et al (17) assessed the QoL for adult patients newly diagnosed with CVD.

Feodor et al (18) assessed CVD prevalence, risk factors and clinical characteristics in the adult population in Romania.

Ianosi et al (19) focused on the treatment of leg teleangectasias in a blind comparative study using Nd:YAG laser vs. sclerotherapy, proving that telangiectasias and reticular veins of the lower extremities can be successfully treated with Nd:YAG laser.

Adverse drug reactions were reported by Iordache et al (20), which showed the incidence and different aspects of skin lesions in contrast-induced chemical hypersensitivity.

Senila et al (21) report a case of progesterone hypersensitivity in a 27-year-old woman with favorable evolution only on topical therapy, the positive clinical outcome being maintained during a subsequent pregnancy and postpartum period.

Fekete et al (22) presented a case of cutaneous leucocytic vasculitis after erlotinib treatment, and the limited number of cases precludes any meaningful interpretation of data regarding Erlotinib-induced cutaneous vasculitis.

Pemphigoid nodularis (PN) is a rare clinical variant of bullous pemphigoid characterized by the presence of nodular prurigo-like lesions and pemphigoid blisters. Vornicescu et al (23) described a case presentation of a patient with pemphigoid nodularis and summarized the reported characteristics of PN.

To conclude, the quality of the papers submitted to the Special Issue of 'Experimental and Therapeutic Medicine' meets the Journal's standards, as it makes fundamental scientific points, thus achieving the proposed aims of the issue. We would like to take this opportunity and thank all the authors for their valuable contribution.

\section{References}

1. Bucur M, Constantin C, Neagu M, Zurac S, Dinca O, Vladan C, Cioplea M, Popp C, Nichita L and Ionescu E: Alveolar blood clots and platelet-rich fibrin induce in vitro fibroblast proliferation and migration. Exp Ther Med 17: 982-989, 2019.

2. Rusu D, Boariu M, Stratul1 ȘI, Bojin F, Paunescu V, Calniceanu H, Surlin P, Roman A, Milicescu S, Caruntu C, et al: Interaction between a $3 \mathrm{D}$ collagen matrix used for periodontal soft tissue regeneration and T-lymphocytes: An in vitro pilot study. Exp Ther Med 17: 990-996, 2019.

3. Grigore O, Mihailescu AI, Solomon I, Boda D and Caruntu C: Role of stress in modulation of skin neurogenic inflammation. Exp Ther Med 17: 997-1003, 2019.

4. Ilie MA, Caruntu C, Lixandru D, Tampa M, Georgescu SR, Constantin MM, Constantin C, Neagu M, Zurac SA and Boda D: In vivo confocal laser scanning microscopy imaging of skin inflammation: Clinical applications and research directions (Review). Exp Ther Med 17: 1004-1011, 2019.

5. Nicolae I, Tampa M, Ene CD, Mitran CI, Mitran MI, Sarbu MI, Matei C, Ene C and Georgescu SR: Correlations between related-purine derivatives and renal disorders in patients with psoriasis vulgaris. Exp Ther Med 17: 1012-1019, 2019.

6. Boca AN, Ilies RF, Saccomanno J, Pop R, Vesa S, Tataru AD and Buzoianu AD: Sea buckthorn extract in the treatment of psoriasis. Exp Ther Med 17: 1020-1023, 2019.

7. Bălănescu AR, Bojincă VC, Bojincă M, Donisan T and Bălănescu SM: Cardiovascular effects of methotrexate in immune-mediated inflammatory diseases (Review). Exp Ther Med 17: 1024-1029, 2019.

8. Surcel M, Huică RI, Munteanu AN, Isvoranu G, Pîrvu IR, Ciotaru D, Constantin C, Bratu O, Căruntu C, Neagu M and Ursaciuc C: Phenotypic changes of lymphocyte populations in psoriasiform dermatitis animal model. Exp Ther Med 17: 1030-1038, 2019.

9. Mihăilă B, Dinică RM, Tatu AL and Buzia OD: New insights in vitiligo treatments using bioactive compounds from Piper nigrum. Exp Ther Med 17: 1039-1044, 2019.

10. Georgescu SR, Tampa M, Mitran MI, Mitran CI, Sarbu MI, Nicolae I, Matei C, Caruntu C, Neagu M and Popa MI: Potential pathogenic mechanisms involved in the association between lichen planus and hepatitis $\mathrm{C}$ virus infection (Review). Exp Ther Med 17: 1045-1051, 2019.

11. Ianosi G, Ianosi S, Calbureanu-Popescu MX, Tutunaru C, Calina D and Neagoe D: Comparative study in leg telangiectasias treatment with Nd:YAG laser and sclerotherapy. Exp Ther Med 17: 1052-1060, 2019.

12. Deleanu D and Nedelea I: Biological therapies for atopic dermatitis: An update (Review). Exp Ther Med 17: 1061-1067, 2019.

13. Nedelea I and Deleanu D: Isolated angioedema: An overview of clinical features and etiology (Review). Exp Ther Med 17: 1068-1072, 2019.

14. Sabău M, Boca AN, Ilies RF and Tătaru A: Potential of high-frequency ultrasonography in the management of atopic dermatitis. Exp Ther Med 17: 1073-1077, 2019.

15. Solomon I, Ilie MA, Draghici C, Voiculescu VM, Căruntu C, Boda D and Zurac S: The impact of lifestyle factors on evolution of atopic dermatitis: An alternative approach (Review). Exp Ther Med 17: 1078-1084, 2019. 
16. Constantin MM, Nita IE, Olteanu R, Constantin T, Bucur S, Matei $\mathrm{C}$ and Raducan A: Significance and impact of dietary factors on systemic lupus erythematosus pathogenesis (Review). Exp Ther Med 17: 1085-1090, 2019.

17. Branisteanu DE, Feodor T, Baila S, Mitea IA and Vittos O: Safety and patient comfort with iodixanol: Impact of chronic venous disease on quality of life: Results of vein alarm study. Exp Ther Med 17: 1091-1096, 2019.

18. Feodor T, Baila S, Mitea IA, Branisteanu DE and Vittos O: Epidemiology and clinical characteristics of chronic venous disease in Romania. Exp Ther Med 17: 1097-1105, 2019.

19. Ianoși SL, Forsea AM, Lupu M, Ilie MA, Zurac S, Boda D, Ianosi G, Neagoe D, Tutunaru C, Popa CM and Caruntu C: Role of modern imaging techniques for the in vivo diagnosis of lichen planus (Review). Exp Ther Med 17: 1106-1112, 2019.

20. Iordache AM, Docea AO, Buga AM, Mitrut R, Albulescu D, Zlatian O, Ianosi S, Ianosi G, Neagoe D, et al: The incidence of skin lesions in contrast media-induced chemical hypersensitivity. Exp Ther Med 17: 1113-1124, 2019.
21. Senila SC, Ungureanu L, Candrea E, Danescu S, Vasilovici A, Cosgarea R and Dionisie V: Progesterone hypersensitivity: Case report with favorable evolution. Exp Ther Med 17: 1125-1127, 2019.

22. Fekete GL and Fekete L: Cutaneous leukocytoclastic vasculitis associated with erlotinib treatment: A case report and review of the literature. Exp Ther Med 17: 1128-1131, 2019

23. Vornicescu C, Senilă SC, Cosgarea R, Candrea E, Pop AD and Ungureanu L: Pemphigoid nodularis - rare presentation of bullous pemphigoid: A case report and literature review. Exp Ther Med 17: 1132-1138, 2019.

This work is licensed under a Creative Common Attribution-NonCommercial-NoDerivatives 4.0 International (CC BY-NC-ND 4.0) License. 